\title{
EFFECT OF CRACK GEOMETRY ON DISLOCATION NUCLEATION AND CLEAVAGE THRESHOLDS
}

L.L. FISCHER and G.E. BELTZ

Department of Mechanical and Environmental Engineering, University of California, Santa Barbara, CA 93106

\begin{abstract}
A continuum model based upon the Peierls-Nabarro description of a dislocation ahead of a crack is used to evaluate the critical mode I loading for dislocation nucleation at the tip of a finite, pre-blunted crack. A similar approach is used to evaluate the critical mode I loading for atomic decohesion. Results are presented for various crack tip root radii (a measure of bluntness), for several crack lengths. It is shown that increasing the crack length increases the critical energy release rate for both material behaviors. Increasing the bluntness of a crack tip always increases the required loading for atomic decohesion but nucleation thresholds are initially decreased by very small increases in crack tip bluntness. Nucleation thresholds are later increased after reaching significant arack tip blunting. Implications for ductile versus brittle competition are discussed by comparing the ongoing competition between these two different material behaviors.
\end{abstract}

\section{INTRODUCTION}

In 1974, Rice and Thomson [1] presented their well-known continuum model for quantifying the competition between dislocation nucleation and atomic decohesion. The basic approach was to evaluate the critical energy release rate of cleavage, given by the Griffith criterion, and to evaluate the critical energy release rate for the emission of a dislocation on a slip plane intersecting the crack tip [2]. The event possessing the lowest critical energy release rate was predicted to be the dominant outcome. Emission of a dislocation implied that the material would continue to emit dislocations thus shielding the stress singularity and preventing further brittle fracture. In contrast, if cleavage was predicted, brittle fracture would persist. The major grievances with the Rice-Thomson model are that it generally only provides a qualitative prediction of the material behavior, it does not address the actual nucleation event but instead calculates the energy to move a well developed dislocation, it uses a vaguely defined core cut-off parameter as the equilibrium formation position, and the model always assumes a perfectly sharp crack.

Rice [3] addressed several of these problems when he presented a model that utilized a Peierls-Nabarro [4,5] dislocation description. Assuming a periodic relationship between the shear stress and slip displacement along a slip plane intersecting the crack tip, this model solves for a distribution of infinitesimal slip displacement that results from far field loading using a nonlinear integral equilibrium equation. Eventually, an instability is reached and the integral equation can no longer be solved for increased applied loading. This instability marks the nucleation of a dislocation from the crack tip. The efforts of many groups have continued to build upon and improve methods for evaluating the critical energy release rate of dislocation nucleation [6,7], but the sharp crack assumption is still universally adopted as the incipient dislocation forms.

Results from various groups in the atomistic community suggest that crack tip geometry can have a substantial quantitative effect on the energies of nucleation and cleavage $[8,9,10,11]$. Physically speaking, an atomically sharp crack is rare, and this is motivation enough to study the effects of crack geometry using continuum analyses. This paper examines the effects of crack tip blunting and crack length on the critical mode I loading for edge dislocation nucleation and atomic decohesion at the tip of a pre-blunted crack.

\section{THEORY}

To study the effect of crack geometry, an elliptically shaped crack is used as shown in Figure 1. Prescribing the major and minor axes of the ellipse defines a crack length and a crack tip root radius of curvature, $\rho=\mathrm{d}^{2} / \mathrm{a}$, at the tip of the ellipse thereby approximating a blunt crack configuration. 


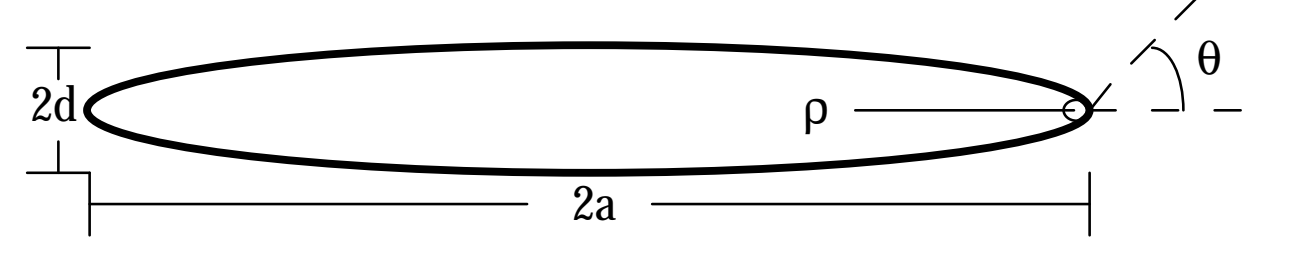

Figure 1. Elliptical crack geometry with crack front and slip plane.

For dislocation nucleation, the elliptical crack is subjected to mode I loading and the equilibrium integral equation is solved for the slip distribution along the slip plane inclined at angle $\theta$.

$$
\tau[\delta(\mathrm{r})]=\sigma_{\mathrm{r} \theta}(\mathrm{r})-\frac{1}{\mathrm{~b}} \int_{0}^{\infty} \sigma_{\mathrm{r} \theta}^{\mathrm{self}}(\mathrm{r}, \mathrm{s}) \frac{\partial \delta}{\partial \mathrm{s}} \mathrm{ds}
$$

The first term in the integral equation represents the periodic relationship between shear stress and slip displacement which is for the sake of simplicity given by the Frenkel sinusoidal function [12],

$$
\tau[\Delta(\mathrm{r})]=\left(\frac{\mu \mathrm{b}}{2 \pi \mathrm{h}}\right) \sin \left(\frac{2 \pi \Delta(\mathrm{r})}{\mathrm{b}}\right)=\frac{\pi \gamma_{\mathrm{us}}}{\mathrm{b}} \sin \left(\frac{2 \pi \Delta(\mathrm{r})}{\mathrm{b}}\right)
$$

where $\tau$ is the shear stress, $\Delta$ is the relative atomic displacement between two atomic planes, $\mathrm{h}$ is the interplanar spacing of those two planes, and $b$ is the Burgers vector. A term known as the unstable stacking energy, $\gamma_{\text {us }}$, is given by $\mu \mathrm{b}^{2} / 2 \pi^{2} \mathrm{~h}$. To obtain the slip displacement occurring on the slip plane as opposed to that occurring between the two atomic planes we use a technique developed by Rice [3]. The extrapolation of $\Delta$ to an imaginary cut halfway between the slipping planes is given by

$$
\delta(\mathrm{r})=\Delta(\mathrm{r})-\frac{\tau[\Delta(\mathrm{r})] \mathrm{h}}{\mu} .
$$

The remaining terms of Equation (1), $\sigma_{\mathrm{r} \theta}$, the applied shear stress along the slip plane, and $\sigma_{\mathrm{r} \theta}{ }^{\text {self }}$, the dislocation self shear stress must be solved for the elliptical crack geometry. In order to solve for the shear stress along the slip plane due to mode I loading a conformal mapping is used where the ellipse of the $\mathrm{z}$ plane will be mapped to a unit circle in the $\zeta$ plane:

$$
\mathrm{z}=\left(\frac{\mathrm{a}+\mathrm{d}}{2}\right) \zeta+\left(\frac{\mathrm{a}-\mathrm{d}}{2}\right) \frac{1}{\zeta}=\mathrm{R}\left(\zeta+\frac{\mathrm{m}}{\zeta}\right)
$$

where $\mathrm{R}=(\mathrm{a}+\mathrm{d}) / 2$ and $\mathrm{m}=(\mathrm{a}-\mathrm{d}) /(\mathrm{a}+\mathrm{d})$. First consider a plane with no crack or hole subject to tensile loading $\sigma$ in the vertical direction. For this case, the principal axes stress components are $\sigma_{\mathrm{yy}}=\sigma$, and $\sigma_{\mathrm{xx}}=\sigma_{\mathrm{xy}}=0$. Assume the complex potentials are of the form:

$$
\Phi(\mathrm{z})=(\alpha+\mathrm{i} \beta) \mathrm{z}, \quad \Psi(\mathrm{z})=(\gamma+\mathrm{i} \delta) \mathrm{z}
$$

where $\mathrm{i}=\sqrt{-1}$ and $\alpha, \beta, \gamma$, and $\delta$ are real. Employing the relationship between the complex potentials and the principal axes stress components:

$$
\begin{gathered}
\sigma_{\mathrm{xx}}+\sigma_{\mathrm{yy}}=2\left(\Phi^{\prime}(\mathrm{z})+\bar{\Phi}^{\prime}(\overline{\mathrm{z}})\right)=\sigma=2(\alpha+\mathrm{i} \beta+\alpha-\mathrm{i} \beta)=4 \alpha \\
\sigma_{\mathrm{yy}}-\sigma_{\mathrm{xx}}+\mathrm{i} 2 \sigma_{\mathrm{xy}}=2\left(\bar{z}^{\prime \prime}(\mathrm{z})+\Psi^{\prime}(\mathrm{z})\right)=\sigma=2(\gamma+\mathrm{i} \delta)=2 \gamma+\mathrm{i} 2 \delta .
\end{gathered}
$$


From Equations 6 and 7, $\alpha=\sigma / 4$, we may arbitrarily set $\beta=0, \gamma=\sigma / 2$ and $\delta=0$. To solve for the image terms due to the elliptical hole, the potentials for no hole are transformed to the $\zeta$ plane and substituted into the following Cauchy integrals where the contour, $\kappa$, lies on the unit circle of the $\zeta$ plane $[13,14]$. These integrals arise from the traction free boundary conditions at the elliptical surface, combined with subsequent analytic continuation arguments.

$$
\begin{gathered}
\Phi_{\mathrm{im}}(\zeta)=\frac{1}{2 \pi \mathrm{i}} \int_{\kappa}\left[\Phi(\zeta)+\frac{1}{\zeta} \frac{\left(\zeta^{2}+\mathrm{m}\right)}{\left(1-\mathrm{m} \zeta^{2}\right)} \bar{\Phi}^{\prime}(\zeta)+\bar{\Psi}(\zeta)\right] \frac{1}{\zeta-\mathrm{x}} \mathrm{d} \zeta \\
\Psi_{\mathrm{im}}(\zeta)=\frac{1}{2 \pi \mathrm{i}} \int_{\kappa}\left[\bar{\Phi}(\zeta)+\zeta \frac{\left(1+\mathrm{m} \zeta^{2}\right)}{\left(\zeta^{2}-\mathrm{m}\right)} \Phi^{\prime}(\zeta)+\Psi(\zeta)\right] \frac{1}{\zeta-\mathrm{x}} \mathrm{d} \zeta-\zeta \frac{\left(1+\mathrm{m} \zeta^{2}\right)}{\left(\zeta^{2}-\mathrm{m}\right)} \Phi_{\mathrm{im}}^{\prime}(\zeta)
\end{gathered}
$$

Using the method of residues and evaluating for the pole at $\zeta=0$ solves for the complex potential image terms. The final complex potentials for a plane with an elliptical hole are given by the sum of the original complex potentials and the image terms. The derivatives of the complex potentials with respect to $\mathrm{z}$ are found using the chain rule and these values are then used as in Equations 6 and 7 to find the stress field. The stress field can then be rotated to solve for the resolved shear stress along the activated slip plane.

Once the stress field for a mode I loaded elliptical hole has been solved, the values can be compared for asymptotic convergence to well known solutions for sharp cracks [15] and circular holes [16]. To compare the atomically sharp crack solution to an extremely sharp elliptical solution we must choose a crack length (the major axis) and a crack tip curvature. For this case we will use $\mathrm{a} / \mathrm{b}=10,000$ and $\rho / b=0.01$ which translates to a minor axis of $2 \mathrm{~d} / \mathrm{b}=20$. Figure $2 \mathrm{a}$ compares the shear stress values for a sharp crack and a sharp ellipse along a slip plane inclined at $\theta=60^{\circ}$. Similarly, Figure $2 \mathrm{~b}$ compares the $\sigma_{\mathrm{r} \theta}$ component for slip along $\theta=60^{\circ}$ for a circular hole and a nearly circular ellipse, using $a / b=10$ and $\rho / b=9.99$.
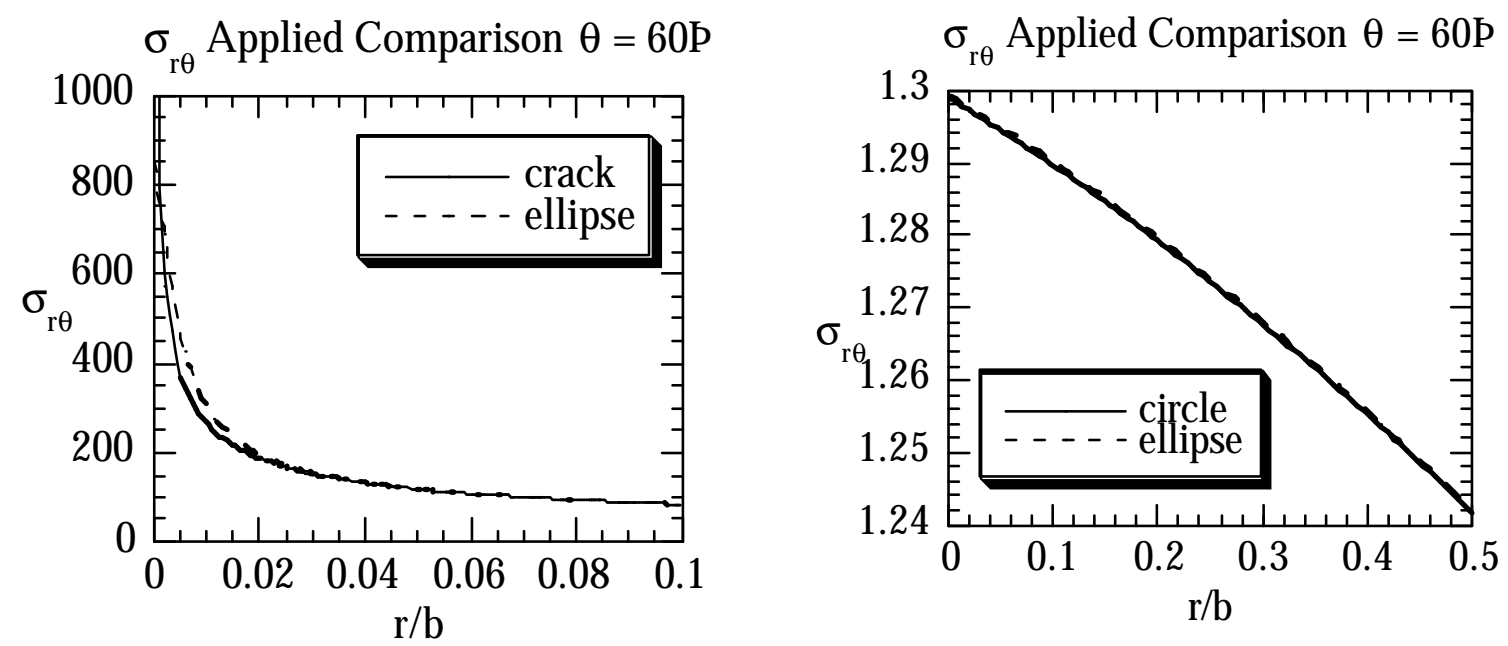

Figure 2 (a) Sharp crack and slender ellipse shear stress comparison. (b) Circular hole and nearly circular ellipse shear stress comparison.

In both cases, the elliptical solutions match the asymptotic solutions very well with the expected exception of the stress singularity at the sharp crack tip. Elliptical solutions will always produce finite stress values at the crack tip.

Solving for the dislocation self stress is done following the exact same steps as used to solve for the applied stress solutions. Of course, there is a different set of original complex potentials for a dislocation in a continuum [13] but finding the image terms and the resolved shear stress is done using exactly the same procedures. The dislocation self stress was also checked for asymptotic convergence to the sharp crack [17] and circular hole [18] solutions and proved once again to make excellent matches. Now that each term in the equilibrium equation has been verified, the integral 
equation itself must be solved for incrementally increasing slip distribution due to incrementally increasing applied loads. The nonlinear integral equation (Equation 1) is rewritten as a summation using a Guass-Chebyshev substitution and is then solved using a numerical procedure similar to that of Beltz [17].

\section{RESULTS}

As previously described, an instability is reached while solving for an incrementally increasing distribution of slip displacement along the slip plane. The stress at which the instability occurs is taken as the critical energy release rate of dislocation nucleation. Figure 3 shows the critical energy release rates for varying values of crack length and crack tip root radius for a slip plane inclined at $\theta=60^{\circ}$. The energy release rates have been normalized by the product $\mu \mathrm{b}$, and the crack lengths and curvatures are normalized by the Burgers vector.

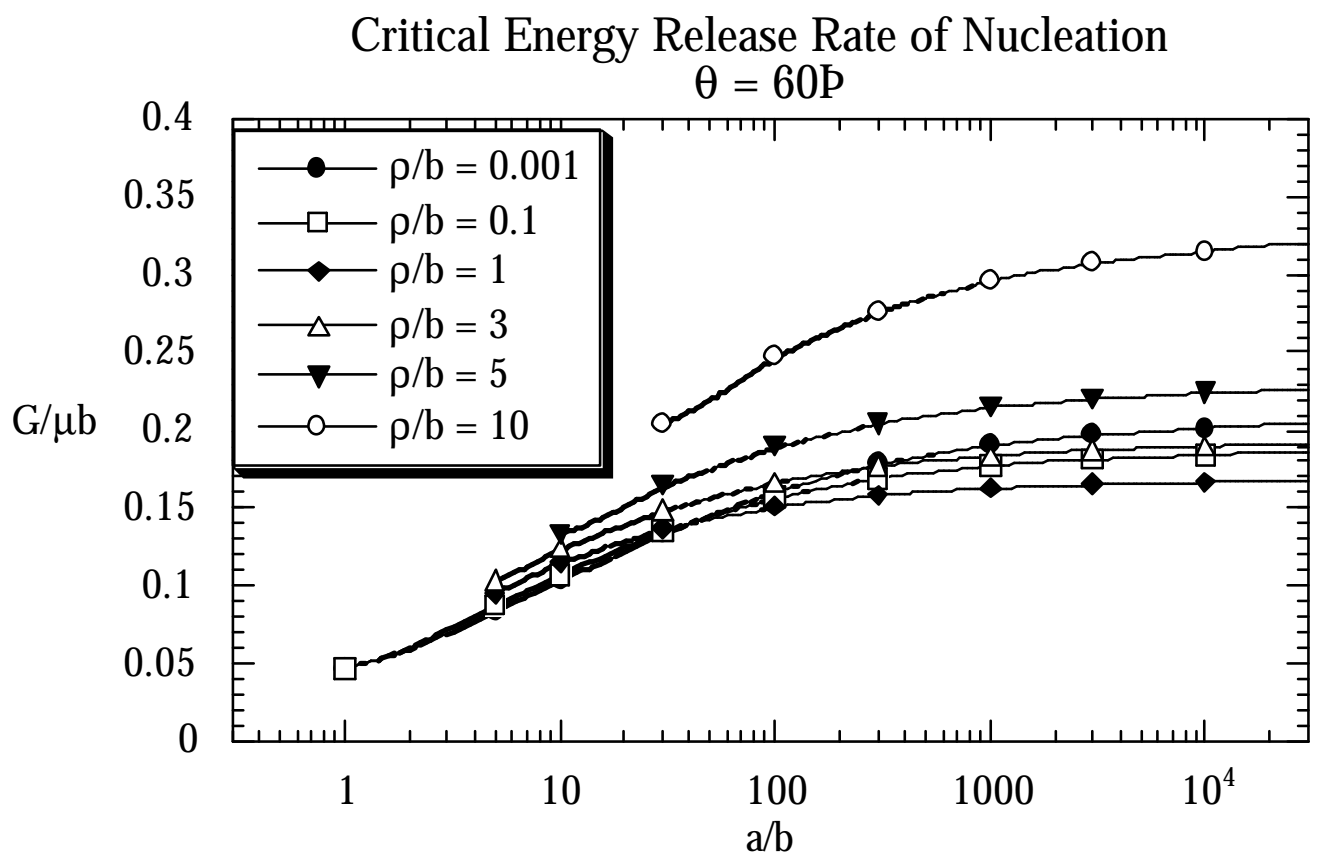

Figure 3. Critical energy release rates of dislocation nucleation for varying $a / b$ and $\rho / b$.

The results clearly show that increasing the bluntness of crack tip at very small radii of curvature decreases the threshold for dislocation nucleation, but at larger radii of curvature, the threshold increases. It is also clear that increasing the crack length increases the threshold for dislocation nucleation.

Solving for the critical energy release rate of atomic decohesion can be done completely by analogy to the dislocation nucleation solution procedure, by assuming an infinitesimal distribution of opening displacement running along the elliptical crack front develops under applied loading. The applied stress and dislocation self stress solutions are now the vertical tensile components of the stress solutions. The opening stress vs. opening displacement relationship is given by

$$
\sigma[\Delta(\mathrm{r})]=\frac{2 \gamma_{\mathrm{s}}}{\mathrm{L}^{2}} \Delta(\mathrm{r}) \mathrm{e}^{\frac{-\Delta(\mathrm{r})}{\mathrm{L}}}
$$

where $\gamma_{s}$ is the surface energy and $L$ is the opening displacement at the peak of the stressdisplacement relationship. This formula must also be expressed in terms of $\delta$, the displacement occurring at the plane of the crack front in order to solve the integral equilibrium equation expression. Varying the crack length and crack tip root radius demonstrates the effect of the two crack tip geometry parameters on the critical energy release rate of atomic decohesion (Figure 4). 


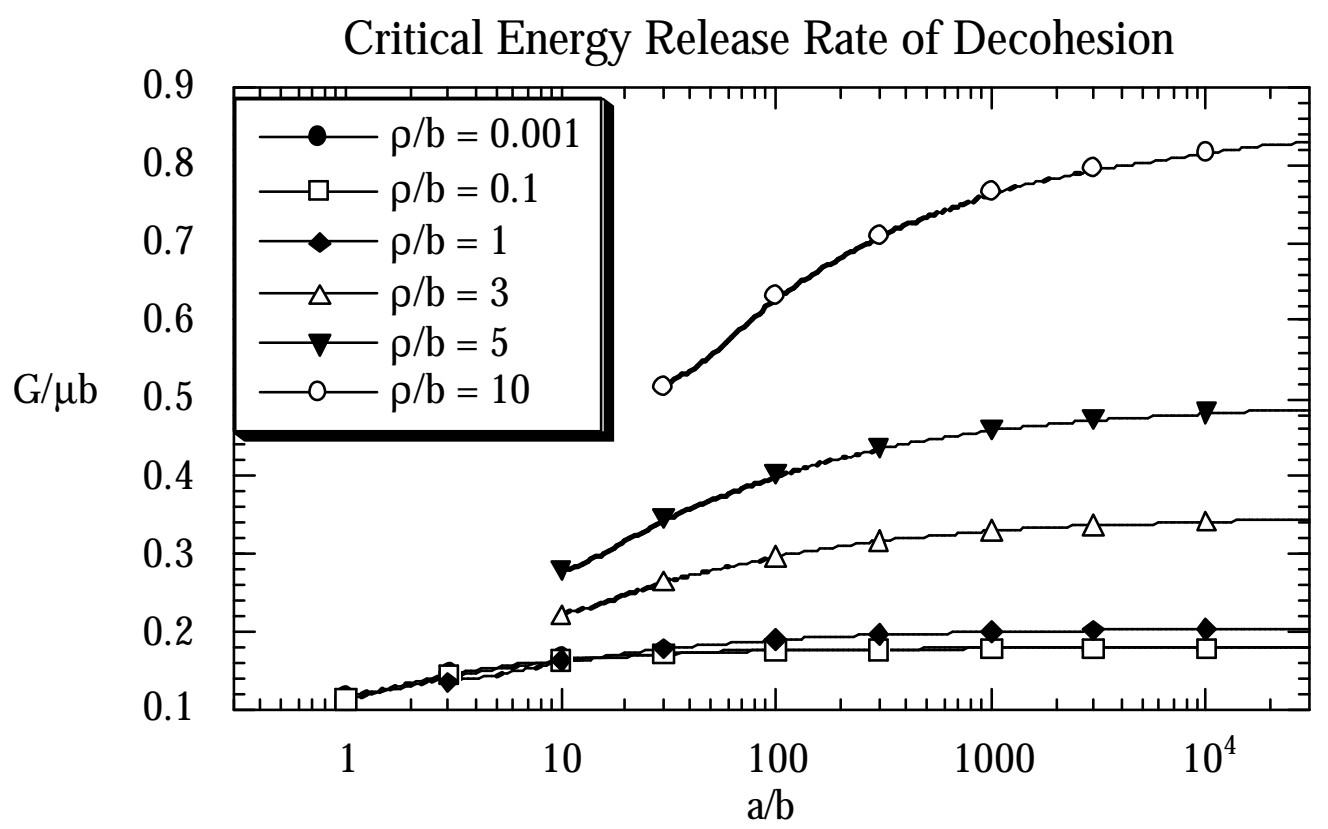

Figure 4. Critical energy release rate of atomic decohesion for varying $a / b$ and $\rho / b$.

The results show that increasing the crack tip root radius increases the threshold for atomic decohesion and increasing the crack length increases the threshold for atomic decohesion.

The results of these two calculations have implications for the theoretical study of ductile vs. brittle material behavior. The results of nucleation and decohesion calculations can be compared on the same plot for a given long crack length. Several material property parameters can be varied to observe different energetic favorabilities. In this case we have used a major axis or crack length of 20,000 Burgers vectors. Comparing the critical energy release rates for varying crack tip curvatures reveals the energetic favorability of the two processes.
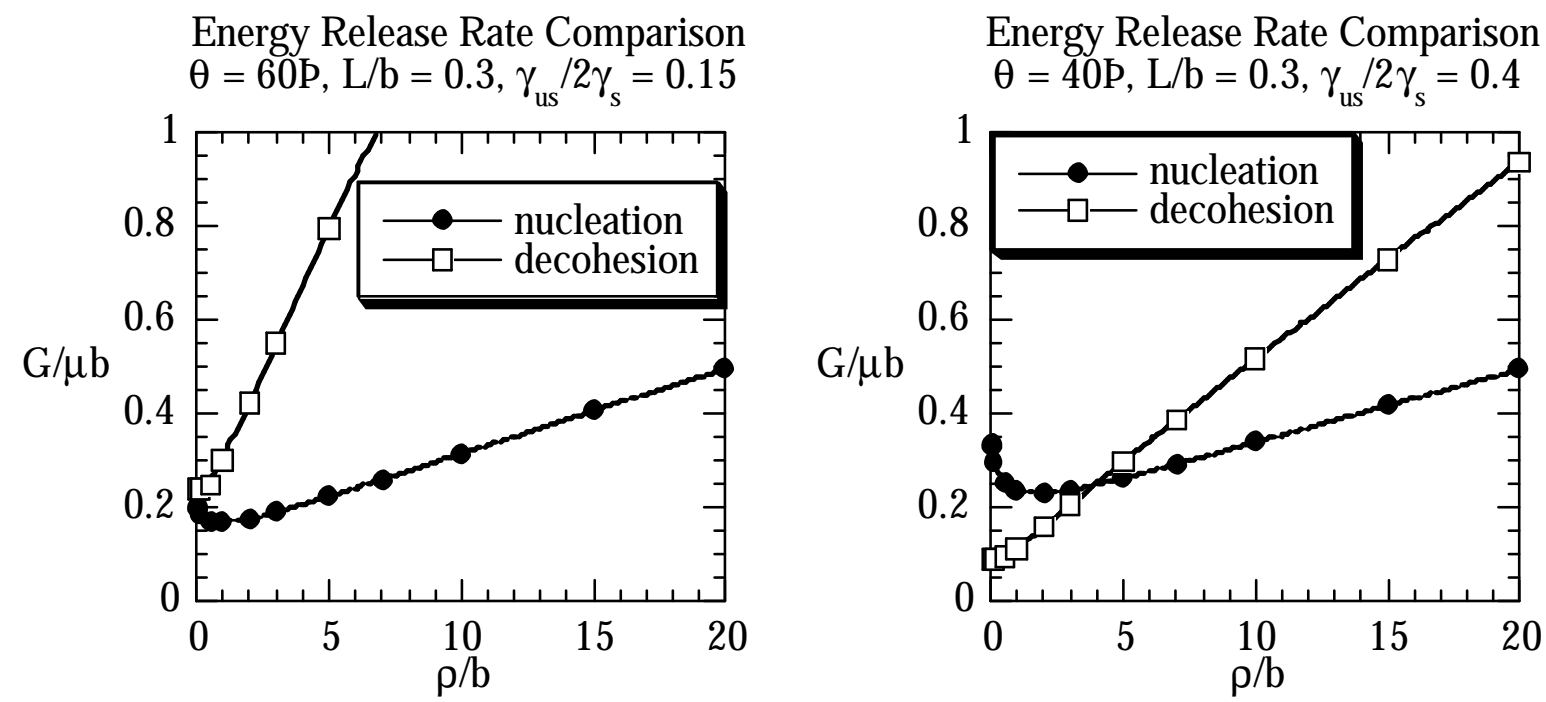

Figure 5. A comparison of dislocation nucleation and atomic decohesion for a long crack length $(\mathrm{a} / \mathrm{b}=10,000)$. (a) Intrinsically ductile (b) Quasi-brittle

In Figure 5a the ratio of the unstable stacking energy to the surface energy is fairly low which is indicative of ductile materials. Furthermore, the slip plane is inclined at $\theta=60^{\circ}$ which is an energetically favorable inclination for dislocation nucleation under mode I loading. Accordingly, the 
graph shows that dislocation nucleation is always favorable to atomic decohesion for all values of crack tip root radius resulting in intrinsically ductile material behavior. In Figure $5 \mathrm{~b}$ the ratio of the unstable stacking energy to the surface energy has been increased to simulate a more brittle material and the inclination of the slip plane has been decreased to $\theta=40^{\circ}$ which is not as conducive to dislocation nucleation for extremely sharp cracks. This combination results in a quasi-brittle material behavior. That is, a fairly sharp crack $(\rho / b<4)$ will continue to be sharp but initially blunt crack configurations may blunt further by dislocation nucleation along the activated slip plane.

\section{CONCLUSIONS}

A continuum model based upon the Peierls-Nabarro description of a dislocation ahead of a crack has shown that crack geometry may have a significant effect on the material fracture behavior. Increasing the length of a crack will increase the thresholds for dislocation nucleation and atomic decohesion while increasing the bluntness of a crack will increase the threshold for decohesion but may decrease or increase the threshold for dislocation nucleation depending on the magnitude of initial blunting of the crack tip. Furthermore, the results for dislocation nucleation and atomic decohesion may be directly compared to ascertain the favorability of the two behaviors for varying crack lengths and crack tip root radii. This observation provides a means of studying ductile versus brittle competition with consideration of crack tip geometry.

\section{ACKNOWLEDGMENTS}

This research was supported by the National Science Foundation under award number CMS9634647 and INT-9707863.

\section{REFERENCES}

1. J. R. Rice and R. Thomson, Phil. Mag. 29, p. 73 (1974).

2. D.D. Mason, Phil. Mag. 39, p. 455 (1979).

3. J. R. Rice, J. Mech. Phys. Solids 40, p. 239 (1992).

4. R. E. Peierls, Proc. phys. Soc. 52, p. 23 (1940).

5. F. R. N. Nabarro, Proc. phys. Soc. 59, p. 256 (1947).

6. J. R. Rice, G. E. Beltz, and Y. Sun, "Peierls Framework for Dislocation Nucleation from a Crack tip," in Topics on Fracture and Fatigue ed. A. S. Argon (New York: Springer-Verlag, 1992), p. 1.

7. G. Xu, A. S. Argon, and M. Ortiz, Phil. Mag. A 72, p. 415 (1995).

8. P. Gumbsch, J. Mater. Res. 10, p. 2897 (1995).

9. P. Gumbsch and G. E. Beltz, Modelling Simul. Mater. Sci. Eng. 3, p. 597 (1995).

10. J. Schiøtz, A. E. Carlsson, L. M. Canel, and R. Thomson, Mat. Res. Soc. Symp. Proc. 409, p. 95 (1996).

11. J. Schiøtz, L. M. Canel, and A. E. Carlsson, Phys. Rev. B 55, p. 6211 (1997).

12. J. Frenkel, Z. Phys 37, p. 572 (1929).

13. V. Vitek, J. Mech. Phys. Solids, 24, p. 67 (1975).

14. N.I. Muskhelishvili, Some Basic Problems on the Mathematical Theory of Elasticity: Fundamental Equations, Plane Theory of Elasticity, Torsion and Bending (Noordhoff, 1975), p. 347.

15. H. Tada, P. C. Paris, and G. R. Irwin, The stress Analysis of Cracks Handbook (Del Research Corporation: St. Louis, 1985), p. 1.4b.

16. S. P. Timoshenko and J. N. Goodier, Theory of Elasticity (McGraw-Hill, Inc.: New York, 1987), p. 90.

17. G. E. Beltz, Ph.D. Thesis, Division of Applied Sciences, Harvard University, Cambridge, Massachusetts, (1992).

18. J. Dundurs and T. Mura, J. Mech. Phys. Solids 12, p. 177 (1964). 